\title{
Diseño de materiales y tecnología. Itinerarios hacia la innovación
}

\author{
Javier RODRÍGUEZ TORRES \\ Universidad de Castilla La Mancha \\ javier.rtorres@uclm.es
}

\begin{abstract}
Resumen:
Nuestra particular hoja de ruta educativa parte de certezas e incertidumbres, de aciertos y errores en el uso y diseño de materiales TIC. Estamos convencidos que la educación con sentido global e incardinado en la cultura de hoy necesita de las ideas, motivos, proyectos y trayectorias profesionales de los que durante algún tiempo llevan poniendo en valor su saber hacer en el día a día.

Desde el acercamiento a la obra de M. Foucault y el análisis de la misma, nos posibilita herramientas de visionarnos y revisarnos educativamente. De igual manera, ha permitido sistematizar como docentes: cómo pensamos, cómo actuamos y cómo nos sentimos (Foucault, 1973; 1975; 1979). Nuestra acción docente no deja de ser una sucesión de prácticas rutinarias, que para Foucault significan un proceso sutil de normalización, producción de individuos (profesores, en nuestro caso) cumplidores de normas, dócil y quizás en proceso de domesticación.

No obstante, el replanteamiento y la reflexión continuada sobre prácticas rutinarias, harán que redescubramos aspectos esenciales de las mismas, que por evidentes necesitan de una nueva forma de mirar, tal vez de unas «gafas socio-culturales» como señalábamos en otro lugar (Rodríguez Torres, 2009), queremos decir, cambiar la forma en que interpretamos, leemos, nos relacionamos con un mundo en constante cambio.
\end{abstract}

Estamos sugiriendo que el uso de las TIC en la educación trascienda los planteamientos instructivos y orientar nuestros esfuerzos docentes hacia planteamientos más comprensivos en torno a las relaciones posibles entre los procesos de cambio social y sus particulares concreciones en los planes de estudio [currículum] (Área 2001; Rayón 1998, 2000). Esta línea de trabajo que venimos desarrollando tiene que ver con la revisión conjunta del profesorado, de su conocimiento pedagógico, tomando como referente su "saber» y su «hacer» en su tarea cotidiana (Cohran - Smith y Lytle, 2002), queremos decir, nuestro objetivo es pasar a una etapa nueva en uso de las TIC que nos permita mejorar los procesos y redescubrir su papel con sentido de globalidad y transversalidad (Rayón y R Torres, 2006)

Palabras clave: TIC; Uso y diseño de materiales; Prácticas docentes; Cambio social; Globalidad

\section{Design of materials and technology. Routes to innovation.}

\begin{abstract}
:
Our particular educational path sheet of certainties and uncertainties of trial and error in the use of ICT and design materials. We are convinced that education with global and incardinated in today's culture need of ideas, motifs, projects and career paths that lead for some time placing value your expertise in the everyday sense.

From the approach to the work of M. Foucault and the analysis thereof, enables us to analyze ourselves educationally tools. Similarly, it has allowed systematize as teachers: how we think, how we act and how we feel (Foucault , 1973, 1975, 1979). Our teaching action continues to be a succession of routine practices that Foucault means a subtle process of standardization, production of individuals (teachers, in our case) standards compliant, docile and perhaps in the process of domestication. However, the continued rethinking and reflection on practice routine will make us rediscover essential
\end{abstract}


aspects of them, for obvious need of a new way of seeing, perhaps a ' socio- cultural glasses' as noted elsewhere ( Rodríguez Torres, 2009), we mean changing the way we play, read, relate to a world of constant change.

We are suggesting that the use of ICT in education transcends the instructional approaches and focus our teaching efforts toward more comprehensive approaches around the possible relationships between the processes of social change and their particular concretions in the curriculum (Area 2001, Rayon 1998, 2000 ). This line of work we have been doing has to do with the joint review of teachers, their pedagogical knowledge, taking as reference the "knowing" and "doing" in their daily task ( Cohran - Smith and Lytle, 2002), we mean our goal is to move to a new stage in the use of ICT to enable us to improve the processes and rediscover its role with regard to global and transversal ( R Rayon and Torres, 2006).

Key Words: ICT; Use and design of materials; teaching practices; social change; Globality.

\section{Referencia normalizada:}

Rodríguez Torres, J. (2014): Diseño de materiales y tecnología. Itinerarios hacia la innovación. Historia y Comunicación Social. Vol. 19. Núm. Especial Marzo. Págs. 675-687.

Sumario: 1. Introducción. 2. Fundamentación teórica. 2.1. Materiales curriculares. Racionalidades. 2.2. Diálogo en la perspectiva lógico - práctica. 3. La realidad que analizamos. 4. Reflexión necesaria para continuar. 5. Bibliografía.

\section{Introducción}

Nuestra particular hoja de ruta educativa parte de certezas e incertidumbres, de aciertos y errores en el uso y diseño de materiales TIC. Estamos convencidos que la educación con sentido global e incardinado en la cultura de hoy necesita de las ideas, motivos, proyectos y trayectorias profesionales de los que durante algún tiempo llevan poniendo en valor su saber hacer en el día a día.

Con la implantación de la LOGSE en la década de 1990 se empieza a utilizar el término de $<<$ materiales curriculares $>>$, para denominar lo que hasta entonces se conocía con el nombre de $<<$ materiales didácticos $>>$. Estos dos términos llevan asociados significados distintos y una orientación educativa diferente, ya que provienen de contextos y modelos pedagógicos distintos.

Los materiales curriculares, también llamados de desarrollo curricular que se emplean como favorecedores del proceso de aprendizaje, son constituyentes del currículo, es por esto, que sean coherentes con todos los demás elementos curriculares.

Por tanto, la elaboración y diseño de materiales curriculares deberá constituir un proceso de relación con otros elementos que va desde los propios objetivos de aprendizaje, pasando por la estimación de necesidades de los alumnos y del medio socio - cultural donde se pongan en práctica. 
Siguiendo a diferentes autores (Cruz, Piñol, 1997), los materiales basados en las TIC tienen en la mayoría de los casos, la potencialidad de un aumento de la motivación en los aprendices, la posibilidad lúdica y la reflexión, tanto de los que aprenden como de los que enseñan.

Con independencia de los materiales curriculares más avanzados tecnológicamente, no podríamos concebir una clase sin su mediación: pizarra, tiza, libros, fotocopias, diapositivas, modelos, ordenadores, dispositivos electrónicos,..

Tras la revisión de distintas definiciones ofrecidas por diferentes autores, desde 1990, Zabala (1990), San Martín (1991), Gimeno (1991), Martínez Bonafé, (1992), Blanco (1994) Parcerisa, (1996), Area (1999) podríamos definir material curricular como cualquier instrumento y medio que proporcione al educador pautas, criterios y ayuda, para la toma de decisiones en el proceso enseñanza - aprendizaje, desde su planificación, intervención y evaluación.

\section{Fundamentación teórica}

\subsection{Materiales curriculares: racionalidades}

Una vez plasmada la perspectiva de diferentes autores sobre el concepto de material curricular, realizaremos una aproximación a las perspectivas más importantes que se producen en el aula, en el momento de clasificar y valorar la función de los materiales curriculares. Primero presentaremos una breve caracterización del papel de los materiales en el marco de una concepción y proceso de creación curricular de corte técnico-racional. Posteriormente analizaremos el otro marco de racionalidad denominado lógico-práctico caracterizado por una orientación más práctica y más abierta, deliberativa y participativa del conjunto de profesionales de la educación y donde el docente tiene una mayor implicación en el diseño y la organización de los materiales curriculares.

\subsubsection{Perspectiva técnico-racional}

Esta perspectiva concentra y centra el interés en la en la fase del diseño curricular. Se cree, que planteando un programa bien estructurado y racionalizado, ofrecerá pocas dificultades para su puesta en práctica. Buscan un material elaborado por expertos, altamente elaborado y que pretende transformar los contenidos del programa curricular en contenidos y actividades para desarrollar en el aula.

Según Allwright (Area, 2002) este modelo parte de dos supuestos básicos respecto del profesorado:

- Un supuesto de deficiencia, en cuanto los medios serían producidos "para proteger a los alumnos de las deficiencias del profesorado, y así asegurar, en 
la medida de lo posible, que el Programa sea cubierto adecuadamente y que los ejercicios sean bien enseñados"

- Un supuesto de diferencia en cuanto que "las habilidades requeridas para la elaboración de materiales son muy diferentes de las exigidas a los profesores de aula"

Podemos decir, por tanto, que los rasgos que caracterizan los materiales desde la propuesta de esta perspectiva son:

- El diseño de medios debe ser responsabilidad de expertos técnicos. No tiene sentido ofrecer participación al profesorado en estas tareas ya que no serían propias de su ámbito de competencias, ni dispone del conocimiento y habilidades necesarias para asumirlas.

- La producción de medios es parte de las tareas del diseño curricular. Estos se elaboran a partir de la lógica y estructura del Programa dando respuesta a demandas emanadas de decisiones administrativas, fundamentadas en mayor o menor medida en principios racionales. La producción de medios, por tanto, apenas tiene en cuenta el conocimiento y necesidades generadas desde las situaciones de desarrollo práctico del currículum.

- Estos materiales presentan una estructura cerrada y poco flexible. Es decir, tienden a prefijar y limitar el tipo de proceso y experiencias de aprendizaje con las que habrá de interaccionar el alumno en el aula.

- A su vez, están concebidos para un modelo prototípico de alumno y contexto cultural. Su naturaleza estructurada y su presupuesto de que todos los alumnos habrán de alcanzar por igual lo mínimo exigido por el Programa favorece que su diseño no tenga en cuenta ni las diferencias entre características y necesidades individuales ni los distintos contextos culturales con sus variantes lingüísticas, económicas, y sociales.

- Existe un exceso de confianza en los medios como la estrategia más eficaz para posibilitar que el profesorado desarrolle prácticas instructivas acorde con el programa innovador.

- El uso que el profesor debiera realizar de los materiales exige de él su aplicación mecánica y fiel en el aula. El material internamente ofrece todo lo que los alumnos pueden necesitar para aprender. Su labor por tanto, se simplifica en el sentido de que no tiene que tomar decisiones relevantes y complejas de planificación de su propio currículum. Su foco de atención debe concentrarse básicamente en tareas de gestión y evaluación del uso del material por los alumnos.

Una vez hemos descubierto los ideales y los rasgos fundamentales de la perspectiva técnico-racional, podemos afirmar que el material por excelencia que más cabida y más peso tiene dentro de esta perspectiva es el libro de texto. 


\subsubsection{Perspectiva lógico-racional}

Esta significa dar mayor entrada, colaboración y capacidad de decisión a los profesores y centros escolares en los mecanismos e instancias de elaboración y distribución de los medios y materiales. En esta orientación o lógica del desarrollo curricular las experiencias prácticas y productos que los profesores realizan con los medios en sus contextos de trabajo son el eje referencial para la producción y difusión de materiales curriculares. Los materiales de este modo se convierten en una estrategia o centro referencial para la movilización de procesos de mejora e innovación educativa y de desarrollo profesional de los docentes.

Esta perspectiva se compone de los siguientes rasgos:

- La producción de medios y materiales no descansa únicamente en instancias técnicas alejadas de la práctica, sino que es el propio profesorado un agente educativo que asume responsabilidades sobre los medios y materiales curriculares que utiliza: bien elaborándolos individual o colectivamente, bien seleccionándolos, bien adaptando materiales previamente existentes tanto del mercado como de otros colegas.

- Se elaboran materiales diversificados territorialmente y adaptados contextualmente a las características de los alumnos. Se tiende a la utilización e integración curricular de los materiales del entorno

- Se desarrollan estrategias y mecanismos para que los materiales elaborados por el propio profesorado (bien de forma individual o bien en grupos o equipos de trabajo) sean difundidos y conocidos entre los propios enseñantes.

- Se facilita y se potencia el intercambio, distribución y elaboración de los materiales en los centros y comarcas...

- Los medios y materiales pueden ser un pretexto para reorganizar y reformular los espacios y ambientes de aprendizaje de los centros escolares en una perspectiva colaborativa. La introducción de nuevas tecnologías en las escuelas (vídeos, ordenadores) por sus características sofisticadas y coste económico no permiten que cada aula disponga de dichos medios. Lo mismo puede ocurrir con otros tipos de materiales (sala de audiovisuales, laboratorio para ciencias de la naturaleza, museo etnográfico sobre la cultura del entorno, biblioteca para el material impreso,...). Ello significa que los profesores deben "compartir" dichos espacios. Y compartir supone organizarse, coordinarse, intercambiar y preparar conjuntamente experiencias y proyectos de utilización conjunta de esos medios y espacios.

\subsection{Diálogo en la perspectiva lógico - práctica}

Dentro de esta perspectiva se observan diferentes visiones, analizaremos las distintas posiciones de diferentes autores hacia los materiales curriculares de esta perspectiva lógico-práctica, observando la visión que de ellos tienen. 
Para empezar a profundizar sobre las diferentes visiones en torno a los materiales curriculares alternativos al libro de texto, vamos a conocer cuál es la función que desde el punto de vista de Manuel Area (1999) deben cumplir estos materiales. Para este autor los materiales curriculares tienen la obligación de que en los procesos de enseñanza se enseñen al alumnado las habilidades necesarias para acceder a las distintas fuentes de información y dominar los lenguajes de representación simbólica. Por tanto defiende que se debe ensañar a aprender. A parte de esta afirmación Manuel Area (1999) también deja claro que "los materiales elaborados desde la lógico práctica tienen la ventaja de ser fruto de la experiencia docente, estar adaptados a los rasgos idiosincráticos del alumno y del contexto desde el cual surgieron. Por tanto presumiblemente serán más efectivos en la labor que desempeñen.

Félix E. González (2001) apuesta también por esta corriente de crear materiales que tengan un sentido en el contexto en el que van a ser desarrollados y argumenta que la función del docente no es una simple transmisión de contenidos (muchas veces sin sentido en el que contexto en el que están siendo desarrollados) para él la acción educativa no debe ni puede limitarse a una mera mediación o transmisión de contenidos carentes de intencionalidad educativa. Por ello ve fundamental que sea el maestro el que cree sus propios materiales para ser utilizados con la intención de conseguir lo que pretende, como además muestra en las siguientes palabras en las que invita a la reflexión de los docentes sobre los materiales utilizados:

cabe destacar el interés y la necesidad que los profesionales de la educación reflexionen y adopten medidas de selección de los materiales curriculares que utilizan como apoyo al proceso de enseñanza aprendizaje.

Para Nieves Blanco (1994) es necesario que haya materiales que puedan ser experimentados y no simples instrumentos que encorseten y uniformicen la práctica. Por tanto, hay que cambiar la concepción de materiales, ya que de lo contrario la autonomía de los docentes quedará reducida a su capacidad para elegir productos comerciales. Mostrando esta autora un claro apoyo a la creación de materiales por parte del profesorado, unos materiales que sean alternativa al libro de texto y que no sea este el único material utilizado en el aula.

Ben-Peretz (Area, 1999) considera de gran importancia los materiales curriculares y son una gran fuente de conocimiento, este autor afirma que:

los materiales curriculares son más complejos y más ricos en sus posibilidades educativas que cualquier lista de objetivos, sean generales o específicos y conllevan más cosas que la expresión de las intención de los autores. (Ben-Peretz, 1995)

Al igual que el autor anterior San Martín (Area, 1994) da una gran importancia a estos recursos, pero incide también en que es importante adoptar un modelo pedagógico para desarrollar cada uno de los materiales que llevemos al aula, y que será la unión de ese material, con el modelo pedagógico lo que dará el éxito o fracaso a lo que desarrollamos en el aula. Considera que el potencial de los materiales curriculares no descansa sólo en sus atributos, en la bondad o calidad de su diseño, este potencial 
alcanza su sentido y virtualidad educativa en el marco del método pedagógico bajo el cual se inserta y se utiliza pedagógicamente.

La visión de Nieves Ledesma ya se dejaba entrever en el apartado anterior en la que manifestaba una opinión en contra de los libros de texto como único medio. Con respecto a la utilización de más materiales curriculares destaca que los profesores deberían seleccionar, adaptar, crear y evaluar los materiales curriculares que van a utilizar en sus aulas además "debe tener una actitud abierta a la innovación".

Tras ver las visiones, opiniones y afirmaciones de los diferentes autores que hemos consultado, nuestro estudio sobre los materiales curriculares continúa analizando, en esta ocasión, la realidad de lo que ocurre en los centros donde se llevan a cabo los procesos de enseñanza-aprendizaje.

\section{La realidad que analizamos:}

Hemos comenzado una investigación con diseño de tipo etnográfico. Se lleva a cabo un estudio longitudinal, durante tres cursos escolares (2012 -13, $2013-14$ y 2014 - 15), de tres centros de la provincia de Toledo, con recursos, materiales y formación suficiente para realizar actividades diferenciadas.

Tabla 1: Características de los centros participantes

\begin{tabular}{|c|c|c|c|}
\hline $\begin{array}{l}\text { Centros educativos } \\
\text { participantes }\end{array}$ & Contexto y ubicación & Tamaño del centro & $\begin{array}{c}\text { Dotación de } \\
\text { ordenadores y medios } \\
\text { audiovisuales }\end{array}$ \\
\hline CEIP A & Barrio urbano & $\begin{array}{c}\text { Dos grupos de } \\
\text { alumnos por nivel } \\
18 \text { unidades (aulas) } \\
\text { y } 20 \text { profesores }\end{array}$ & $\begin{array}{l}\text { Rincón del ordenador } \\
\text { en E. Infantil } \\
\text { Aula Althia }\end{array}$ \\
\hline CEIP B & $\begin{array}{l}\text { Barrio urbano } \\
\text { Centro concertado }\end{array}$ & $\begin{array}{c}\text { Dos grupos de } \\
\text { alumnos por nivel } \\
18 \text { unidades (aulas) } \\
\text { y } 19 \text { profesores }\end{array}$ & $\begin{array}{l}\text { Aula de informática } \\
\text { (16 puestos), } 1 \text { pizarra } \\
\text { digital por ciclo }\end{array}$ \\
\hline CEIP C & $\begin{array}{l}\text { Entorno rural alejado } \\
\text { de zona metropolitana }\end{array}$ & $\begin{array}{c}\text { Un grupo de } \\
\text { alumnos por nivel } \\
9 \text { unidades (aulas) y } \\
12 \text { profesores }\end{array}$ & Aula Althia \\
\hline
\end{tabular}

Los interrogantes que centraron el trabajo son:

1. Cuestiones organizativas entorno a los recursos

2. Planificación de las TIC en el proceso de enseñanza: metodología, evaluación y experiencias de innovación

3. Actitud del profesorado 
Teniendo en cuenta esto interrogantes, el trabajo de investigación se propuso los siguientes objetivos:

- Conocer los materiales más utilizados y frecuentes en los centros escolares en la planificación del proceso de enseñanza.

- Reconocer prácticas innovadoras: la apertura a nuevas metodologías, implementación de materiales alternativos, favorecer el aprendizaje colaborativo,..

- Obtener en profundidad la visión del profesorado de los centros con respecto a materiales curriculares.

Durante este primer curso las tareas realizadas han sido:

- Contextualización de los centros y conocimiento

- Inmersión en el campo de estudio: análisis de necesidades, actitudes, debilidades, amenazas, fortalezas y oportunidades.

- Recopilación de prácticas novedosas y satisfactorias para el profesorado

- Análisis de prácticas

Para llevar a cabo el trabajo sobre el uso de materiales curriculares en los centros, se realizaron en cada centro análisis DAFO en el que se detallan las debilidades, amenazas, fortalezas y oportunidades que puede tener el material curricular. Para extraer conclusiones se diseñó un formulario anónimo que fue pasado en cada claustro.

Tal y como se preveía antes que poner el práctica el cuestionario, el material más utilizado, con gran protagonismo y con gran presencia en las aulas resulta ser el libro de texto. La media del total de los encuestados detalla un uso del libro de texto en un $72 \%$ del total de las sesiones. Dato muy significativo y que nos da algunas pistas de la visión de los docentes de dichos centros y de la dependencia que tienen hacia este material.

En el siguiente gráfico se muestra la relación agrupada del uso que todos los encuestados hacen de dicho material.

\section{Reflexión necesario para continuar}

Nuestra acción docente no deja de ser una sucesión de prácticas rutinarias, que para Foucault significan un proceso sutil de normalización, producción de individuos (profesores, en nuestro caso) cumplidores de normas, dócil y quizás en proceso de domesticación.

No obstante, el replanteamiento y la reflexión pertinaz y continuada sobre nuestra rutina, harán que redescubramos aspectos esenciales de nuestra práctica docente, que por evidentes necesitan de una nueva forma de mirar, tal vez de unas "gafas 
socio-culturales" como señalábamos en otro lugar (Rodríguez Torres, 2011), queremos decir, cambiar la forma en que interpretamos, leemos, nos relacionamos con un mundo en constante cambio, nos estamos refiriendo explícitamente a:

1. El alumno (en su diversidad) protagonista absoluto y esencial, motor de nuestro quehacer docente

2. La necesidad de buscar respuestas para atender y dar cauce a su diversidad y complejidad en un mundo en constante cambio (los materiales curriculares)

3. La comprensión de nuestra acción por qué, para qué, qué, cómo. ... Esto nos ha exigido la realización de "viajes" de ida y vuelta de nuestra práctica a la teoría y, en ocasiones, a la inversa.

Por otro lado y desde nuestros planteamientos, deudores de Paulo Freire, la adquisición de competencias digitales no es sólo un problema de manejo de mecánicas y uso de artefactos, sino que debe constituir un aprendizaje profundo y global, es decir, la adquisición de recursos intelectuales para interactuar, tanto con la cultura existente, como para recrearla de un modo crítico que haga llegar a la Comunidad Educativa a emanciparse, en definitiva, a ser autónomos, más ciudadanos y más libres.

Estamos sugiriendo que la integración de las TIC en la educación obligatoria tenga que trascender los planteamientos instructivos y orientar nuestros esfuerzos docentes hacia planteamientos más comprensivos en torno a las relaciones posibles entre los procesos de cambio social y sus particulares concreciones en el currículum (Área 2001; Rayón 1998, 2000).

Esta línea de trabajo que venimos desarrollando tiene que ver con la revisión conjunta del profesorado, de su conocimiento pedagógico, tomando como referente su "saber" y su "hacer" en su tarea cotidiana (Cohran - Smith y Lytle, 2002), queremos decir, nuestro objetivo es pasar a una etapa nueva en la integración de las TIC que nos permita mejorar los procesos y redescubrir su papel en el currículum con sentido de globalidad y transversalidad (Rayón y R Torres, 2006)

En otro orden de cosas, las TIC, consideradas como materiales curriculares, son medios empleados en los procesos de enseñanza - aprendizaje y, por tanto, mediadores de la cultura. Siguiendo a Apple (1989), los materiales curriculares tienen una función añadida, "subjetiva", el control del curriculum y de la enseñanza. Jurjo Torres (1989) llega a denominarlos "productos políticos" al reproducir concepciones ideológicas de grupos dominantes.

A este respecto, A Bautista (1998) advierte de la necesidad de concretar las finalidades y contenidos que deben sustentar la integración de la tecnología en la escolarización. Para este autor, la reflexión sobre los modelos de desarrollo tecnológico, los intereses que promueven el uso de las tecnologías en los distintos ámbitos sociales y el debate sobre los principios éticos que deben regir la aplicación y uso de la tecnología en la sociedad son dimensiones esenciales que justificarían y determinarían los contenidos a incorporar en la educación de los alumnos. Dimensiones que, a nuestro 
juicio, dotarían a los proyectos educativos, orientados a la integración de las TIC en la escuela, de un carácter verdaderamente educativo e innovador. Y sin embargo, ni la integración, ni el potencial formativo de estos recursos ha sido debatido atendiendo a las implicaciones socioculturales y éticas que tienen para la sociedad y los individuos.

Así conviene advertir que el debate relativo a por qué, para qué, qué tecnologías incorporar, cómo hacerlo y en qué dirección le ha sido usurpado al docente, a quien se le ha colocado en situación de usuario y receptor de una toma de decisiones de expertos y técnicos que deciden por ellos. Porque otro rasgo definitorio de la política educativa es la tecnificación y burocratización de la formación permanente del profesorado, que se desarrolla bajo un plan jerárquico en el que los técnicos y expertos en informática y telecomunicaciones toman decisiones en función del mercado.

Nadie discute que las TIC suponen un cambio, pero casi nadie habla de que éste tiene que ir acompañado de una nueva semiótica de la escuela, queremos decir, las aulas deben concebirse como entornos de trabajo.

En la actualidad, asociamos tecnología con innovación. Pero, compartimos con otros autores, que la introducción de cualquier medio tecnológico en los centros educativos pasa ineludiblemente por disponer de una actitud convencida y favorable por parte de los docentes, como también, por una adecuada formación de los mismos para su correcta incorporación en su práctica cotidiana (Cabero, Duarte y Barroso, 1997). Por tanto, como decíamos con anterioridad, en este nuevo contexto tecnológico es necesario que el profesorado cambie su actitud y su rol con respecto a la enseñanza.

La innovación en el contexto educativo se ha venido asociando a renovación, cambio y a mejora. Aunque toda innovación supone renovación, cambio y mejora; no toda renovación, cambio y mejora es innovación.

Fullan (1992) ha explorado las complejas relaciones entre la mejora de la escuela y el cambio, sirvan sus palabras para ilustrar esta idea:

conseguir la mejora de la escuela depende de la comprensión del problema que implica el cambio en la práctica y el desarrollo de las estrategias correspondientes para producir reformas ventajosas (p. 7)

Desde nuestra propia práctica y experiencia compartida, la innovación supone una actitud de superación de rutinas que van a beneficiar a quienes más lo necesitan (los alumnos, sujetos de atención diferenciada). Esta actitud supone en los docentes:

- Reflexión continuada sobre la propia práctica

- Autonomía docente

- Crítica

- Cambio que se produce por una exploración sistemática de la realidad. 
En los grupos humanos, cada uno de nosotros tiene una forma especial de pensar, de sentir y actuar. Esta variabilidad junto a las diferentes capacidades, necesidades, intereses, ritmos de maduración, condiciones socio - culturales, entre las más importantes, conforman cada uno de los grupos sociales en los que desarrollamos nuestra vida con una distribución normal, y, en ocasiones, en los extremos aparecen sujetos con diferencias más evidenciadas. En la escuela, grupo humano, existen tales circunstancias, aquí se debe ofrecer una atención específica, en caso necesario, durante el tiempo de la escolarización. Es decir, estamos entendiendo la diversidad del alumnado en su amplio sentido, sin restricciones.

Como consecuencia esencial la diversidad es, desde nuestro punto de vista, un elemento enriquecedor $\mathrm{y}$, por consiguiente, clave para la planificación de todos los elementos que intervienen en el proceso de enseñanza - aprendizaje. Sin embargo, no es suficiente que los alumnos con determinadas necesidades estén "integrados" en las aulas, sino que deben participar plenamente en la vida escolar y social de la misma.

Entendemos que una escuela inclusiva es aquella que:

- Atienda la multiciplidad de variables ambientales, frente a planteamientos centrados en las diferencias de los alumnos

- Procure cambios metodológicos y organizativos, de modo que la atención a alumnos con dificultades beneficie a todos los demás

- Diseñe ambientes escolares que estimulen la participación de todos, promoviendo las relaciones sociales y el éxito escolar (Echaita et al, 2006)

Completando el concepto de escuela inclusiva compartimos con Fernando Arreaza [1] que la escuela que apuesta por enseñar lo importante, renuncia a utilizar el saber cómo instrumento de selección del alumnado y pone los medios para hacer posible que la calidad educativa alcance a todos sin exclusión, para ello nos propone la siguiente imagen que refleja de forma gráfica las características de escuela inclusiva.

\section{Bibliografía}

[1] http://lascompetenciasbasicas.wordpress.com/ (visitada 6 de julio de 2009) AINSCOW, M (2001). Desarrollo de escuelas inclusivas. Ideas, propuestas y experiencias para mejorar las instituciones escolares. Madrid. Narcea.

APPLE, M. (1989): Maestros y textos. Barcelona. Paidós

AREA, M. (1999). Los materiales curriculares en el contexto de los procesos de diseminación y desarrollo del currículum. En Diseño, desarrollo e innovación del curriculum. Madrid : Síntesis.

AREA, M. (2002). Los medios y el curriculum escolar. Web docente de Tecnología Educativa . 
ARTILES, R. M. (1994). Libros de texto y desarrollo del currículo en el aula. Universidad la Laguna.

BAUTISTA GARCÍA-VERA, A (1998). Tecnología, mercado y gobernabilidad: un trinomio interactivo en la enseñanza a finales del segundo milenio. Revista Complutense de Educación, 9 (1), pp. 29-46.

BAUTISTA GARCIA-VERA, A. (2007). Alfabetización tecnológica multimodal e intercultural. Revista de Educación, 343. pp. 589 - 600

BOOTH, T. \& AINSCOW, M. (2002). Guía para la evaluación y mejora de la educación inclusiva. Desarrollando el aprendizaje y la participación en las escuelas. Madrid. CSIE y Consorcio Universitario para la Educación Inclusiva.

BERNABÉ,I Y ADELL, J. (2006). Los libros de texto de la escuela en red. Perspectiva CEP (11), 21-33.

BLANCO, N. (1994) Materiales curriculares: los libros de texto. En Teoría y desarrollo del curriculum (págs. 175-185). Universidad de Málaga.

CABERO, J.; DUARTE, A. y BARROSO, J. (1997). La piedra angular para la incorporación de los medios audiovisuales, informáticos y nuevas tecnologías en los contextos educativos y el perfeccionamiento del profesorado. EDUTEC, 8.

CARBONELL, J. (2001). La aventura de innovar. Madrid. Morata

COCHRAN - SMITH, M Y L. LYTE, S. (2002). Dentro/Fuera. Enseñantes que investigan. Madrid AKAL. Educación Pública.

ECHEITA SARRIONANDIA G. (2006) Educación para la inclusión o educación sin exclusiones. Madrid. Narcea.

FREIRE, P. (1990) [1985]. La naturaleza política de la educación. Cultura, poder y liberación. Barcelona. Paidós.

FREIRE, P. (1998) [1969]. La educación como práctica de la libertad. México. S. XXI.

FOUCAULT, M. (1970). La arqueología del saber. México. Siglo XXI.

FOUCAULT, M. (1979). Microfisica del Poder. Madrid. La Piqueta

FOUCAULT, M. et. al. (1991). Espacios de poder. Genealogía del Poder, no 6, Madrid. Ediciones de La Piqueta

FULLAN, M (1992). The role of the principal and school improvement. Successful school improvement. Buckingham, Philadelphia. Open University Press

FULLAN, M. y HARGREAVES, A. (1997) ¿Hay algo por lo que merezca la pena luchar en la escuela?. Morón (Sevilla). M.C.E.P.

FULLAN, M. (2002). Los nuevos significados del cambio en la educación. Barcelona. Octaedro.

GARCÍA, J. J. (2012). Material práctico para su aplicación en instituciones socioeducativas.

GARRIDO, J. M. (2001). El papel de los materiales curriculares en la intervención educativa. Revista de Educación (3), 221-229.

GIMENO SACRISTÁN, J. (1998). Poderes inestables en educación. Madrid. Morata

GIMENO SACRISTÁN, J. (2002). Educar y convivir en la cultura global. Madrid. Morata. 
GIMENO SACRISTÁN, J. (2005). La Educación que aún es posible. Madrid. Morata.

GIMENO SACRISTÁN, J .(2005). El currículum como texto de la experiencia. De la calidad de la enseñanza a la del aprendizaje. En La educación que aún es posible. Morata S.L.

GONZÁLEZ, F. E. (2001). Criterios para valorar materiales curriculares: una propuesta de elaboración referida al rendimiento escolar. Revista Complutense de Educación, 12 (1), 179-212.

HARGREAVES, A (2005). Profesorado, cultura y postmodernidad. (Cambian los tiempos, cambia el profesorado). Madrid. Morata

JOHNSTON, M. (1997) El significado de la colaboración: Más allá de las diferencias culturales. Kikiriki Cooperación Educativa, $n^{\circ} .46$

JOHNSON, D. W.; JOHNSON, R. T. y HOLUBEC, E. J. (1999) El aprendizaje cooperativo en el aula. Barcelona. Paidós.

LEDESMA, N. (1997). Materiales curriculares y formación del profesorado. Revista Electrónica Interuniversitaria de Formación del profesorado (1).

LOZANO, C. (2013). Las mujeres las grandes ausentes de los libros de texto. Pikara Magazine.

MORÍN, E. (2000) La Mente bien ordenada. Barcelona, Seix-Barral

TABOADA, M. B. (2008) Libros de texto en la enseñanza de la lengua: Recorridos críticos y construcción de identidades. Universidad Autónoma de Entre Ríos.

RAYÓN RUMAYOR, L y RODRÍGUEZ TORRES, J. (2006). La necesaria $<<$ voz>> del docente para la integración curricular de las TIC. $O G E, \mathrm{n}^{\circ}$ 4, julio, pp. 23 - 26

RODRÍGUEZ TORRES, J (2011): Discursos, poder y saber en la formación permanente: La perspectiva del profesorado sobre la integración curricular de las TIC. Editorial Académica. Madrid.

RODRÍGUEZ TORRES, J. (2012) Evaluación inicial de competencias en un programa de diversificación curricular, punto inicial para la reflexión de nuestra práctica y replanteamientos de elementos básicos del curriculum. Quaderns Digitals / Quaderns número 71 monográfico Estrategias Didácticas Inclusivas

TORRES SANTOMÉ, X (1998). El curriculum oculto. Madrid. Morat 